\title{
Fast single-step least-squares reverse-time imaging via adaptive matching filters in beams
}

\author{
Qiancheng Liu, Member, IEEE, Yongming Lu, and Hao Zhang
}

\begin{abstract}
Least-squares reverse time migration (LSRTM) is a powerful tool in seeking broadband-wavenumber reflectivity images. It produces better images over reverse-time migration (RTM) at the expense of computational cost. The Hessian effect can be measured in the image domain with the pointspread function (PSF). We here try to measure the Hessian effect in the data domain with a so-called trace-spread function (TSF). The difference between PSF and TSF is that the former originates from $L^{T} L$ in the image domain while the latter from $\mathbf{L L}^{T}$ in the data domain. By comparing the TSFs with their original corresponding traces (or beams), we can design adaptive matching filters for preconditioning to alleviate the Hessian effect. However, the full TSF matrix is costly. In this paper, we propose a multi-scale solution, which first has a diagonal approximation to $\mathbf{L L}^{T}$ in beams, and then handle the full sub-matrix composed of the one-beam traces using the Sherman-Morrison formula. The preconditioned beams are superimposed into a "deblurred" data for remigration. Through synthetic and real data examples, we see that (i) single-step data-domain LSRTM can yield deblurred RTM images via adaptive matching filters; (ii) the beam-by-beam consideration outperforms the trace-by-trace one.
\end{abstract}

Index Terms-Adaptive matching filter, Fast inversion

\section{INTRODUCTION}

$\mathbf{R}$ EVERSE-time migration (RTM) has been the geophysical workhorse in imaging complicated structures without dip limitation for over decades [1], [2], with extended applications to ground-penetrating radar imaging [3], [4], electromagnetic imaging [5], and medical imaging [6], [7]. However, as an adjoint operator rather an inverse one [8], RTM images suffer from the blurring effect caused by Hessian, which can be measured in the image domain as the point-spread function (PSF) [9]. Least-squares reverse-time migration (LSRTM) can deblur the RTM images by minimizing the residual between the predicted and observed data. [10] first proposed the concept of the least-squares migration (LSM), which afterward catches the wide attention from academia and industry. LSM is first applied to Kirchhoff migration [11], [12], and then phase-shift migration [13], [14] and one-way wave-equation migration [15], [16], and now RTM. Here, we mainly focus on LSRTM.

Based on the two-way wave equation, LSRTM has no dip limitation in inverting for the broadband-wavenumber reflectivity model. [17] image the ocean-bottom data with LSRTM.

Q. Liu is now with Department of Geosciences, Princeton University, NJ 08544, USA, and formerly with the Department of Physical Sciences and Engineering, King Abdullah University of Science and Technology, Thuwal, Saudi Arabia (e-mail: qiancheng.liu@kaust.edu.sa)

Y. Lu is with the Department of Earth and Space Sciences, Southern University of Science and Technology, Shenzhen, China (luym@ @ustc.edu.cn).

$\mathrm{H}$. Zhang is with the Institute of Geomechanics, Chinese Academy of Geological Sciences, Beijing, China (zhanghao@geomech.ac.cn).
[18] exploit source-encoding to improve the computational efficiency of LSRTM. [19] jointly consider the reflection waves and free-surface multiples to increase imaging illumination. [20] propose a cross-correlation objective function based practical LSRTM solution. [21] formulate LSRTM in a generalized matrix form. [22] propose an efficient step-length formula for the cross-correlative LSRTM. [23] introduce a prestack approach in the cross-correlative LSRTM. [24] illustrate that the formulation of the exact adjoint operator to the forward modeling can improve the performance of LSRTM.

Preconditioning, which may help to make cheaper the LSM computation cost, can be implemented either in the image or the data domain. In the image domain, we usually consider the approximations to the Hessian [25]. [26] utilizes the nonstationary matching filtering to approximate the inverse Hessian. [9] approximates the Hessian using PSFs. [16] improves the computational efficiency of target-oriented PSFs by using a phase-encoding strategy. The data-domain approaches may be more likely to be stationary and economical when compared with the depth-domain approaches. Several methods, such as deconvolution [27] and automatic gain control (AGC) [28], are proposed. However, these methods are mainly derived from the viewpoint of digital signal processing.

From the viewpoint of wave-equation migration and demigration, [29] propose a single-step data-domain LSRTM by comparing the observed data with the demigrated data to output a deblurred data by adaptive matching filters for remigration. Their approach, however, takes the deblurring process trace-by-trace under the diagonal approximation to the $\mathbf{L L}^{T}$ operator. In practice, the seismic waves are more likely to propagate with the spatially bandlimited effects in beams rather than in rays [30]. The beam-by-beam consideration may improve the performance of the existing single-step datadomain LSRTM. To verify this, similar to the concept of PSF, we propose a concept of trace-spread function (TSF). The difference between PSF and TSF is that the former is governed by $\mathbf{L}^{T} \mathbf{L}$ while the latter by $\mathbf{L} \mathbf{L}^{T}$. We use the TSF to evaluate the spreading effect of one trace or one bundle of traces (in beams) in the demigrated data over the migration/demigation processes. Under the beam approach, we consider the $\mathbf{L L}^{T}$ operator at two levels. At the beam level, we use the diagonal approximation to $\mathbf{L} \mathbf{L}^{T}$. At the level of traces in one beam, we still fully consider the sub-matrix from $\mathbf{L L}^{T}$ by using the Sherman-Morrison formula [31]. A similar approach in handling geophysical inverse problems can be found in [32]. The processed beams are then merged into a "deblurred" common-shot dataset for remigration.

In this paper, we first briefly review the conventional it- 
erative LSRTM. Then, we derive the theory of the singlestep data-domain LSRTM. Afterward, under the concept of TSFs, we compare the TSF performances of the trace-by-trace operation and beam-by-beam operation, and propose beambased adaptive matching filters to single-step LSRTM in the data domain. Finally, we test our methods on the Marmousi synthetic data and one 2D marine data.

\section{THEORIES AND METHODS}

\section{A. Review of iterative LSRTM}

We here only focus on the acoustic case with constant density. Iterative LSRTM mainly consists of three steps: demigration (Born modeling), migration (RTM) and linearized optimization. There is no dip limitation in LSRTM because both the demigration and migration operators are based on the two-way wave equation. For simplicity, we represent the demgiration and migration operators in matrix-vector notation in the following.

As stated in Appendix A, we express the Born modeling as

$$
\mathbf{L m}=\mathbf{d},
$$

where $\mathbf{L}$ is the demigration operator denoted in Eq. (A-3), $\mathbf{m}$ the reflectivity and $\mathbf{d}$ the seismograms obtained from $\mathbf{m}$. The size of $\mathbf{L}$ is $N_{m}$ by $N_{r}$, with $N_{m}$ being the size of the model and $N_{r}$ the number of the receivers. For stacked RTM images, Eq. (1) is overdetermined. Here, we parameterise the logarithmic reflectivity as $\mathbf{m}=\delta v / v$, with $\delta v$ being the velocity perturbation and $v$ the background velocity [33]. Under the Born approximation, given the true reflectivity, $\mathbf{d}$ can be the observed data. In seismic imaging, only with $\mathbf{d}$ and migration velocity at hand, we attempt to image the underground structures with the adjoint operator [33]. However, in practice, the RTM profile is inferior to the true reflectivity model due to the imperfectness of the adjoint operator. To overcome this imperfectness and gain an inverted reflectivity model of better quality, we adopt a least-squares inversion with the following least-squares misfit:

$$
E(\mathbf{m})=\frac{1}{2}\|\mathbf{L m}-\mathbf{d}\|_{2}^{2} .
$$

LSRTM is an ill-posed problem. A regularization may not play a big role for noise-free or less noisy data, but will be important for noisy data. The normal equation of Eq. (2) reads

$$
\mathbf{L}^{T} \mathbf{L m}=\mathbf{L}^{T} \mathbf{d},
$$

where $\mathbf{L}^{T}$ is the adjoint operator of $\mathbf{L}$. However, we notice that the solution to Eq. (3) involves the data-misfit Hessian inverse $\left(\mathbf{L}^{T} \mathbf{L}\right)^{-1}$, the full form of which is prohibitive to store or calculate for practical applications.

RTM simply has the adjoint image [11] as

$$
\mathbf{m}_{m i g}=\mathbf{L}^{T} \mathbf{d}=\mathbf{L}^{T} \mathbf{L} \mathbf{m},
$$

from which we notice that $\mathbf{L}^{T} \mathbf{L}$, the Hessian, acts as a blurring operator upon $\mathbf{m}$, resulting in $\mathbf{m}_{m i g}$. It is impossible to account for the Hessian matrix for practical problems fully. As a proper solution, iterative LSRTM chooses to seek the inverted image via linearized inversion by using the gradient optimization methods, such as steepest descent or conjugate gradient [12]. The iterative LSRTM is costly in computation. Therefore, we try to look for a powerful but inexpensive preconditioner for fast inversion.

\section{B. Deblurring in the data domain}

Eq. (4) suggests the possibility to deblur RTM in the image domain if we can get the inverse of $\mathbf{L}^{T} \mathbf{L}$. Some authors make approximations to $\left(\mathbf{L}^{T} \mathbf{L}\right)^{-1}$ in different manners, such as local layered media assumption [34], diagonal approximation [25], non-stationary matching filter [26] and PSFs [9], [16]. Here, we attempt to deblur in the data domain.

We start from the definition of a novel misfit function

$$
F\left(\mathbf{d}^{*}\right)=\frac{1}{2}\left\|\mathbf{L}^{T} \mathbf{d}^{*}-\mathbf{m}\right\|_{2}^{2} \text {. }
$$

Unlike the misfit function in Eq. (2), Eq. (5) attempts to find the optimal data $\mathbf{d}^{*}$ to minimize the model misfit between the reflectivity model $\mathbf{m}$ and the adjoint image $\mathbf{L}^{\mathbf{T}} \mathbf{d}^{*}$. Deriving Eq. (5) with respect to the unknown $\mathbf{d}^{*}$ yields its normal equation as

$$
\mathbf{L} \mathbf{L}^{T} \mathbf{d}^{*}=\mathbf{L} \mathbf{m} .
$$

For single-shot RTM images, Eq. (1) becomes underdetermined such that Eq. (6) makes sense. Assuming a generalised invertible approximation to $\left(\mathbf{L} \mathbf{L}^{T}\right)^{-1}$, given $\mathbf{L m}=\mathbf{d}$, we approximately have

$$
\mathbf{d}^{*} \approx\left(\mathbf{L} \mathbf{L}^{T}\right)^{-1} \mathbf{d}
$$

For estimation of $\left(\mathbf{L L}^{T}\right)^{-1}$, we first obtain the raw RTM image $\mathbf{m}_{m i g}$ from the observed data $\mathbf{d}$ using Eq. (4). Then, we have the demigrated data $\tilde{\mathbf{d}}$ from $\mathbf{m}_{m i g}$ as follows

$$
\tilde{\mathbf{d}}=\mathbf{L} \mathbf{m}_{m i g}=\mathbf{L} \mathbf{L}^{T} \mathbf{d}
$$

Under a generalized matrix inverse, we can approximately determine the inverse of $\mathbf{L} \mathbf{L}^{T}$ by

$$
\left(\mathbf{L} \mathbf{L}^{T}\right)^{-1}=(\mathbf{d} \otimes \tilde{\mathbf{d}})(\tilde{\mathbf{d}} \otimes \tilde{\mathbf{d}}+\mu \mathbf{I})^{-1},
$$

where $\otimes$ denotes the outer product operator, $\mathbf{L L}^{T}$ is a square matrix. Although $\mathbf{d}$ and $\tilde{\mathbf{d}}$ are column vectors, $\mathbf{d} \otimes \tilde{\mathbf{d}}$ and $\tilde{\mathbf{d}} \otimes \tilde{\mathbf{d}}$ are square matrices. In addition, because the calculation of Eq. (9) involves matrix inverse, we add a stabilizer $\mu \mathbf{I}$, with $\mathbf{I}$ being an identity matrix, to the diagonals of $\tilde{\mathbf{d}} \otimes \tilde{\mathbf{d}}$.

Finally, let us go back to Eq. (7). We can obtain the singlestep inversion result if we precondition the observed data $\mathbf{d}$ with the estimated adaptive matching filter $\left(\mathbf{L L}^{T}\right)^{-1}$. This filter has no specific pattern but is determined by the pair of observed and demigrated data.

\section{Data-domain deblurring in bandlimited beams}

It is expensive to solve the problem in Eq. (9) entirely, especially for the 3D case. [29] make it a simplified solution by only considering its diagonal entries, leading to a traceby-trace operation. The trace-by-trace consideration resembles that of ray-tracing [35], which is the asymptotic approximation. The seismic signals, however, cannot travel downwards 


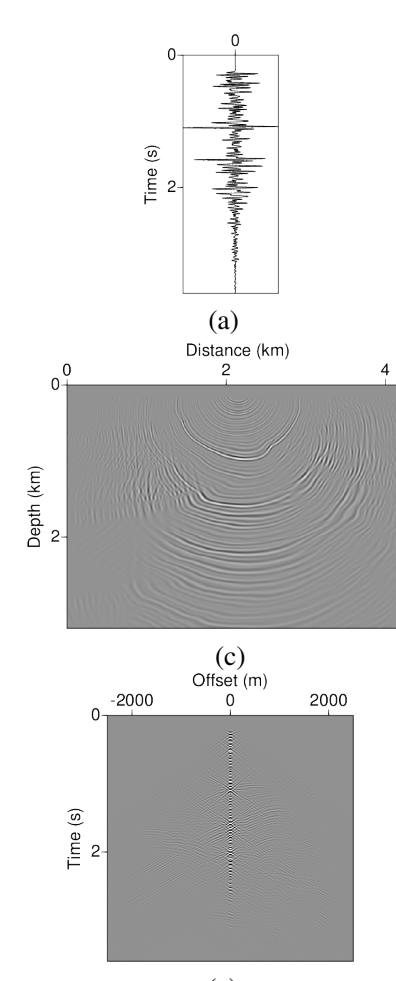

(e)

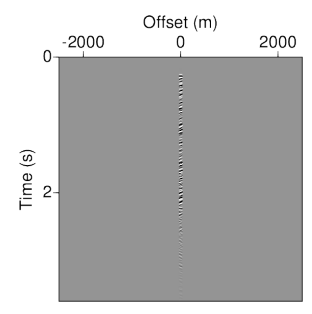

(b)

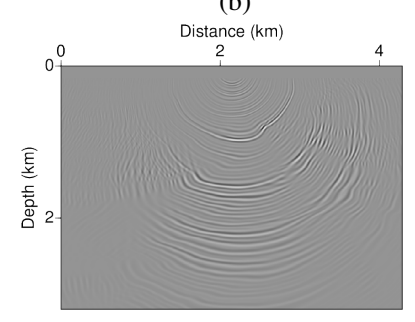

(d)

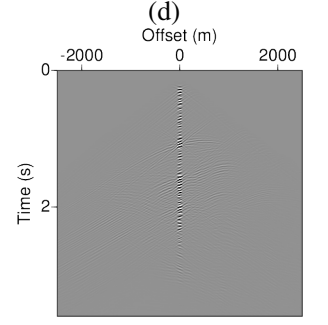

(f)

Fig. 1. Trace-spread function (TSF) testings for (a) one trace, and (b) one beam. Shown in (c) and (d), (e) and (f) are their responding images, and demigrate data, respectively. We can see that the energy distribution in (f) is more focused with fewer artifacts than that in (e).

to the image domain, and then go back to the data domain in rays accurately, due to the following relationship:

$$
\left[\begin{array}{c}
\tilde{d}_{1} \\
\tilde{d}_{2} \\
\vdots \\
\tilde{d}_{N_{r}}
\end{array}\right]=\left[\begin{array}{cccc}
L_{1} L_{1}^{T} & L_{1} L_{2}^{T} & \cdots & L_{1} L_{N_{r}}^{T} \\
L_{2} L_{1}^{T} & L_{2} L_{2}^{T} & \cdots & L_{2} L_{N_{r}}^{T} \\
\vdots & \vdots & \ddots & \vdots \\
L_{N_{r}} L_{1}^{T} & L_{N_{r}} L_{2}^{T} & \cdots & L_{N_{r}} L_{N_{r}}^{T}
\end{array}\right]\left[\begin{array}{c}
d_{1} \\
d_{2} \\
\vdots \\
d_{N_{r}}
\end{array}\right]
$$

in which $N_{r}$ denotes the number of receivers. From Eq. (10) we can see that even with only one trace of $\mathbf{d}$, its contribution will spread over all the traces in the demigrated data $\tilde{\mathbf{d}}$. As shown in Eq. (8), the demigrated data $\tilde{\mathbf{d}}$ from the RTM image provide a viable way to measure in the data domain the "blurring effect" caused by the adjoint operator. Luckily, even after the $\mathbf{L L}^{T}$ process, the seismic signals still have the spatially band-limited feature, which we will show below. Similar to the idea of the point-spread function (PSF) in the image domain, we propose a concept of trace-spread function (TSF) in the data domain. The difference between PSF and TSF is that the former extracts information from $\mathbf{L}^{T} \mathbf{L}$ while the latter from $\mathbf{L L}^{T}$. For demonstration, in the left panel of Fig. 1, we first migrate one single trace in Fig. 1a to get its RTM image in Fig. 1c, from which we generate the demigrated data shown in Fig. 1e. Shown from Fig. 1e is that similar to the PSF, the TSF also has the band-limited feature in space. The primary energy of the demigrated data distributes around the emitting observed data-trace. We can see that: (i) it is expensive to output every TSF for $\mathbf{L} \mathbf{L}^{T}$ because

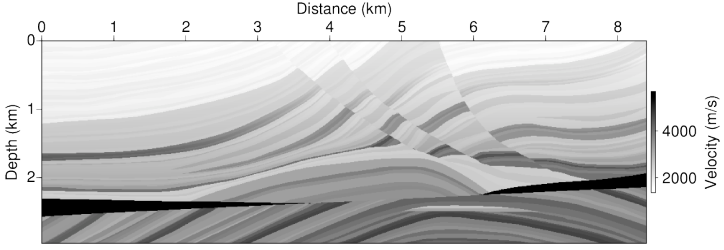

(a)

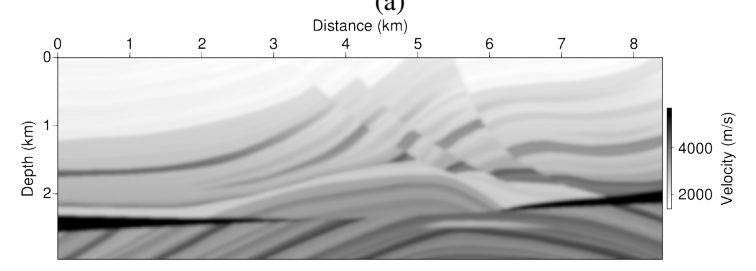

(b)

Fig. 2. Marmousi benchmark: (a) true velocity for the generation of the observed data, (b) migration velocity.

each needs a migration/demigration pair; (ii) the trace-by-trace consideration is not enough to fully solve the inverse problem in Eq. (9) due to the energy-spreading and trade-offs in Fig. 1e.

Inspired by the ideas from the Gaussian-beam migration [30], which honors the bandlimited characteristics of seismic waves in space, we take the seismic data not trace-by-trace but beam-by-beam instead. To get the beams, we decompose the data with a series of Gaussian windows. According to [30], the initial width of the Gaussian beam in the data domain depends on the frequency and local velocity, and the results are not sensitive to this choice as long as the initial width (in the data domain) is around the value:

$$
w_{G}=\bar{v} / f_{m},
$$

where $\bar{v}$ is the average of the velocities around the receivers, and $f_{m}$ is the central frequency of the seismic data. The right panel of Fig. 1 illustrates the beam consideration in the TSF testing. We first migrate the beam data in Fig. 1b to get the RTM image in Fig. 1d, from which we get the demigrated beam data in Fig. 1f. By comparing Figs. 1d and 1f, we find that the bands of the two beams are nearly the same, with weak energy spreading out. By cross-comparison between Figs. 1e and 1f, we find that the TSF of the latter contains much weaker off-diagonals. These comparisons suggest that we may handle Eq. (10) in a beam-by-beam diagonal approximation as follows

$$
\left[\begin{array}{c}
\widetilde{b}_{1} \\
\widetilde{b}_{2} \\
\vdots \\
\widetilde{b}_{N_{b}}
\end{array}\right]=\left[\begin{array}{cccc}
\Lambda_{1} \Lambda_{1}^{T} & 0 & \cdots & 0 \\
0 & \Lambda_{2} \Lambda_{2}^{T} & \cdots & 0 \\
\vdots & \vdots & \ddots & \vdots \\
0 & 0 & \cdots & \Lambda_{N_{b}} \Lambda_{N_{b}}^{T}
\end{array}\right]\left[\begin{array}{c}
b_{1} \\
b_{2} \\
\vdots \\
b_{N_{b}}
\end{array}\right],
$$

where $\mathbf{b}=\mathbf{W d}$ and $\tilde{\mathbf{b}}=\mathbf{W} \tilde{\mathbf{d}}$, with $\mathbf{W}$ being the Gaussianwindowing operator, $N_{b}$ denotes the number of decomposed Gaussian beams in the data domain. Physically, the $\boldsymbol{\Lambda} \boldsymbol{\Lambda}^{T}$ operator is the same with $\mathbf{L L}^{T}$ but neglecting the weak tradeoffs. We here employ $\boldsymbol{\Lambda} \boldsymbol{\Lambda}^{T}$ for distinction because we can make a diagonal approximation under the beam-by-beam consideration.

The formulation in Eq. (12) is equivalent to a multi-scale solution to Eq. (10). At the level of the bandlimited beam, we 


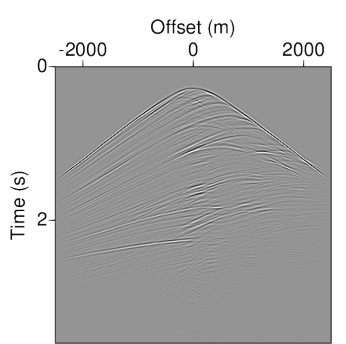

(a)

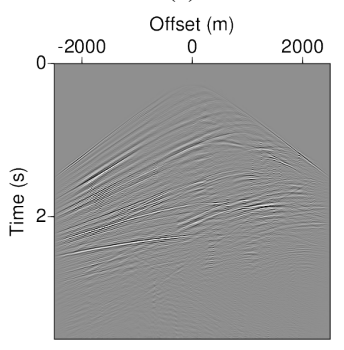

(c)

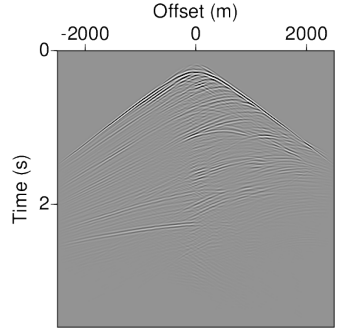

(b)

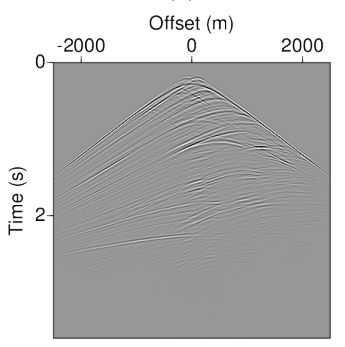

(d)
Fig. 3. (a) observed data, (b) the demigrated data from RTM image, (c) deblurred data via adaptive matching filter with beam-by-beam consideration, (d) the demigrated data from single-step LSRTM image. We observe that (d) is closer to (a) than (b).

can have a solution of diagonal approximation. Each beam contains a bundle of traces within the Gaussian window. At the level of traces, we still need to solve an equation similar in form to Eq. (10). This time, however, the size for $\mathbf{L L}^{T}$ dramatically reduces from $N_{r} \times N_{r}$ to $N_{b} \times N_{b}$, with $N_{r}$ being the number of seismic traces and $N_{b}$ being the number of seismic beams. Obviously, we have $N_{r} \gg N_{b}$.

We solve Eq. (12) in beams. When it comes to the traces in one beam, each entry in Eq. (12) should be vectors, although we express them in the scalar form as a whole. To avoid confusion, for example, given $\widetilde{b}_{1}=\Lambda_{1} \Lambda_{1}^{T} b_{1}$, we rewrite them into vectors $\widetilde{\mathbf{x}}=\mathbf{A} \mathbf{A}^{T} \mathbf{x}$, with $\widetilde{\mathbf{x}}=\widetilde{b}_{1}, \mathbf{x}=b_{1}$ and $\mathbf{A}=\Lambda_{1}$. To get the inverse of $\mathbf{A} \mathbf{A}^{T}$, following Eq. (9), we have

$$
\left(\mathbf{A} \mathbf{A}^{T}\right)^{-1}=(\mathbf{x} \otimes \widetilde{\mathbf{x}})(\widetilde{\mathbf{x}} \otimes \widetilde{\mathbf{x}}+\mu \mathbf{I})^{-1} .
$$

Eq. (13) is solved in the frequency domain. $\mu=$ $\frac{\alpha}{N_{\omega} * N_{b}}\left(\sum|\widetilde{\mathbf{x}}|^{2}\right)$ is an adaptive stabilizer related to the average of the summed diagonals of $\widetilde{\mathbf{x}} \otimes \widetilde{\mathbf{x}}$ over all effective frequency points, with $0<\alpha<1$ and $N_{\omega}$ being the number of frequency points. According to the Sherman-Morrison formula [31], [32], we can expand the term in Eq. (13) as

$$
(\widetilde{\mathbf{x}} \otimes \widetilde{\mathbf{x}}+\mu \mathbf{I})^{-1}=\frac{1}{\mu}\left(\mathbf{I}-\frac{\widetilde{\mathbf{x}} \otimes \widetilde{\mathbf{x}}}{\mu+\langle\widetilde{\mathbf{x}}, \widetilde{\mathbf{x}}\rangle}\right),
$$

in which $\langle$,$\rangle denotes the inner product operator. The final form$ of Eq. (13) reads

$$
\left(\mathbf{A A}^{T}\right)^{-1}=\frac{\mathbf{x} \otimes \widetilde{\mathbf{x}}}{\mu}\left(\mathbf{I}-\frac{\widetilde{\mathbf{x}} \otimes \widetilde{\mathbf{x}}}{\mu+\langle\widetilde{\mathbf{x}}, \widetilde{\mathbf{x}}\rangle}\right) .
$$

For each $\mathrm{x}$ and $\widetilde{\mathrm{x}}$ pair, we directly precondition the $\mathrm{x}$ with its corresponding $\left(\mathbf{A A}^{T}\right)^{-1}$ over all the effective frequency points via matrix-vector product: $\left(\mathbf{A A}^{T}\right)^{-1} \mathbf{x}$. Note that for each frequency point, $\mathbf{x} \otimes \widetilde{\mathbf{x}}$ is a rank-one matrix, so we try to make the matrix full-rank by adding a stabiliser. However, the

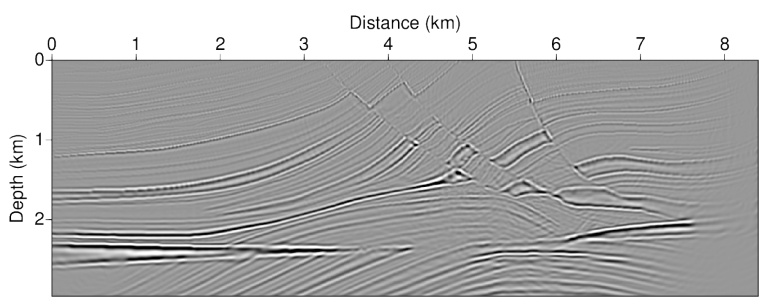

(a)

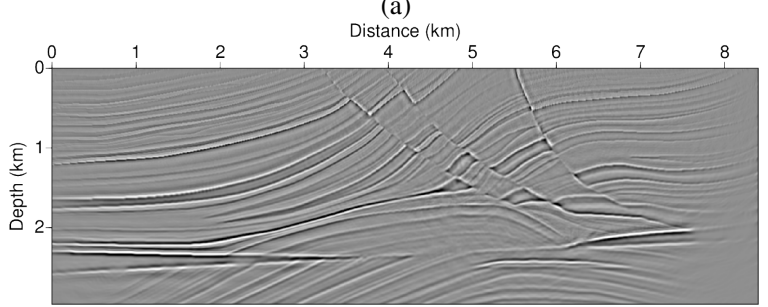

(b)

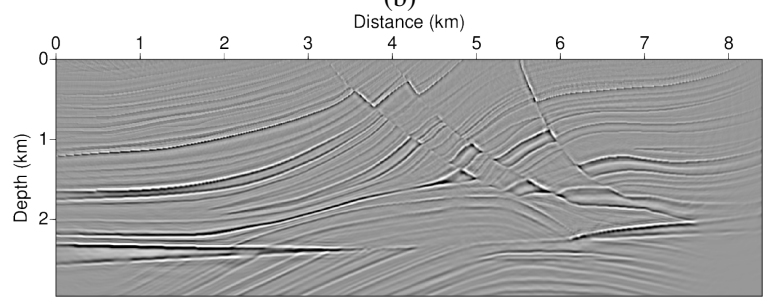

(c)

Fig. 4. (a) stacked RTM image after source illumination, (b) and (c) stacked single-step data-domain LSRTM images with trace-by-trace and beam-bybeam considerations, respectively. We can see that both (b) and (c) are better than (a). With the beam-by-beam consideration, (c) is even slightly better than (b).

inverse of the full-rank matrix is still represented with a rankone matrix. It is worth mentioning that we are trying to have an approximated solution, rather than an exact solution, to the inverse of $\mathbf{L L}^{T}$ in Eq. (10). A similar solution can be found in [32]. With more information gained from the neighboring traces during the data-domain deblurring, the beam approximation is expected to perform better than the diagonal one. This beam operation is memory-effective, because we only need an additional matrix of size $N_{b} \times N_{b}$ for $\left(\mathbf{A A}^{T}\right)^{-1}$. We then superimpose all the preconditioned beams to form a "deblurred" data $\left(\mathbf{L} \mathbf{L}^{T}\right)^{-1} \mathbf{d}$ for the remigration operation. The computational cost of the deblurring in the data domain is negligible compared to that of the wavefield modeling. After the data-domain deblurring is done, we band-pass the data after preconditioning to avoid boosting noises at the low and high frequency ends.

\section{NUMERICAL EXAMPLES}

In this section, we test our methods on the Marmousi synthetic data and one 2D marine data.

\section{A. 2D Marmousi Benchmark}

We test our methods using the 2D Marmousi synthetic data. We perform the modeling with an $O\left(d t^{2}, d x^{4}\right)$ staggered-grid finite-difference stencil [36]. We generate the observed data using the true velocity model in Fig. 2a, with 40 shots evenly spaced at $200 \mathrm{~m}$. The source function is a $20-\mathrm{Hz}$ Ricker 


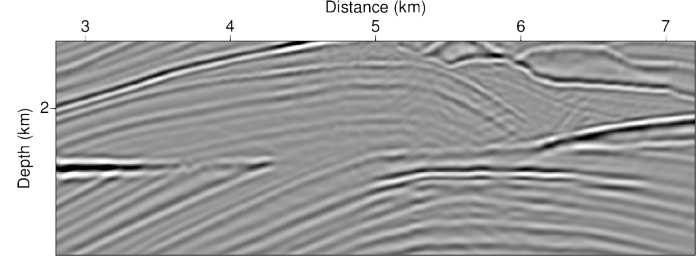

(a)

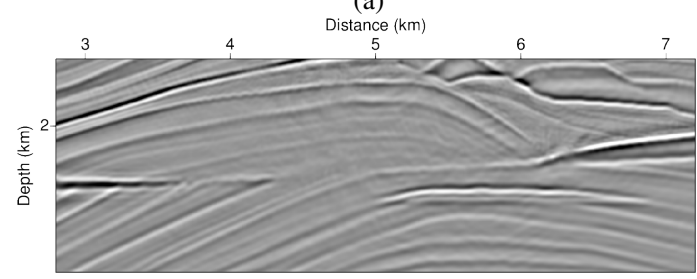

(b)

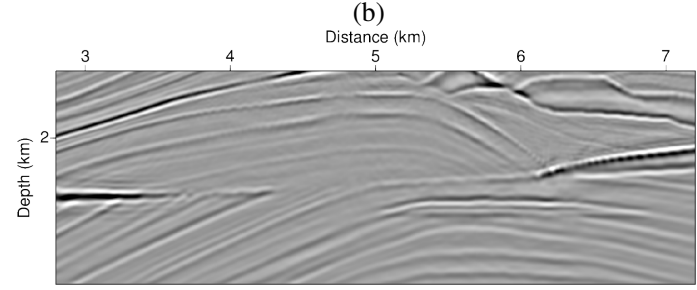

(c)

Fig. 5. (a), (b) and (c) zoom-in views from Figs. 4a, 4b and 4c, respectively We can see that (c) has the best imaging quality among them, with better imaging resolution and less imaging artifacts.

wavelet. We acquire the observed data with a fixed-spreading acquisition geometry of 501 receivers spacing at $10 \mathrm{~m}$. The time duration for the common-shot gathers (CSGs) is $4 \mathrm{~s}$. The direct waves are muted in the CSGs. We obtain the migration velocity in Fig. 2b by blurring the true velocity with a $400 \mathrm{~m}$ Gaussian smoother, removing the high-wavenumber features.

Fig. 3a shows one CSG of the observed data at shot-point $4000 \mathrm{~m}$. We first migrate the observed data to get the RTM image, and the demigrate from the RTM image to get the demigrated data, as shown in Fig. 3b. Afterward, we consider the data-domain deblurring in Eq. (9). Its detailed implementation is shown by Eqs. (12) and (13) in the following steps: (i) decompose the observed and demigrated data into beams; (ii) beam-by-beam deblurring in the data domain; (iii) merge the deblurred beams into one dataset; (iv) remigrate the dataset to image domain. The beamwidth in this example is $75 \mathrm{~m}$. Fig. 3c shows the deblurred CSG.

Fig. 4a shows the stacked RTM image, Fig. $4 \mathrm{~b}$ the singlestep LSRTM image with the trace-by-trace consideration, Fig. $4 \mathrm{c}$ also shows the single-step LSRTM image but with the beam-by-beam approach. For a detailed comparison, we select out three zoom-in views around the Marmousi traps, which is of interest for industrial oil and gas exploration, as shown in Fig. 5. By comparing Figs. 5a, 5b, and 5c, we can see that the single-step LSRTM images have superior imaging qualities, such as sharper and more balanced reflectors, over the RTM image. By comparing Figs. 5b and 5c, we can find the beamby-beam consideration in the single-step LSRTM outperforms the trace-by-trace approach. The difference in the performance of two LSRTMs is due to that the seismic waves propagate more likely as beams with bandlimited effects rather than rays,

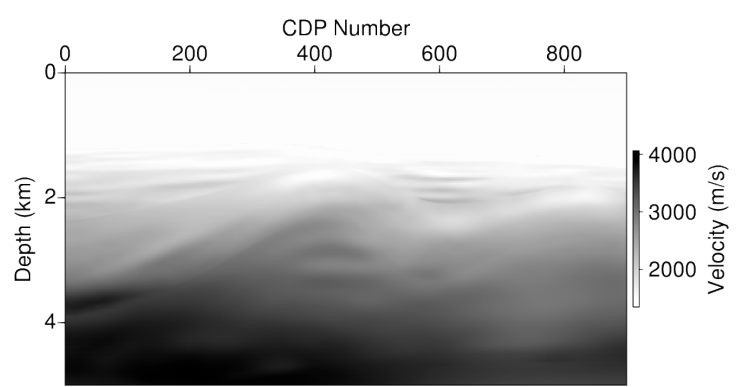

Fig. 6. Migration velocity for the 2D marine data.

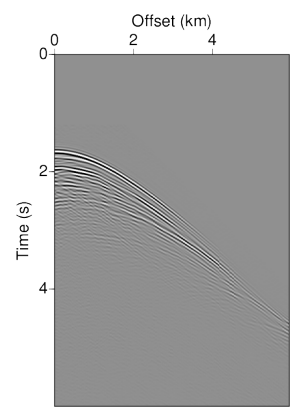

(a)

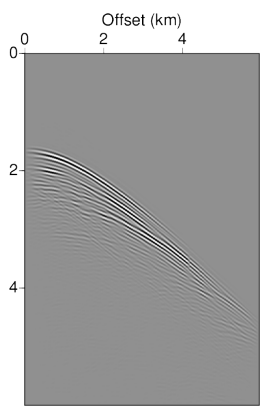

(b)

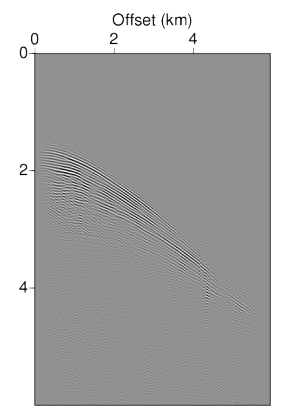

(c)
Fig. 7. 2D marine datasets: (a) observed data, (b) demigrated data and (c) deblurred data for remigration.

as suggested by Figs. 1 .

\section{B. 2D Marine dataset}

We furthermore investigate the application of our methods on a real 2D marine dataset. An acquisition cable record the dataset using 460 receivers at an interval of $12.5 \mathrm{~m}$, with offset ranging from $100 \mathrm{~m}$ to $5750 \mathrm{~m}$. There are 240 CSGs with an evenly shot interval of $37.5 \mathrm{~m}$. The recording duration is $8 \mathrm{~s}$, and the sampling rate is $2 \mathrm{~ms}$. The dominant frequency of this data we use here is around $10 \mathrm{~Hz}$. Fig. 6 shows the migration velocity model for this dataset. The workflow for this dataset is similar to the Marmousi testing above, but with a half-integral correction for the 2D Green's function as an industrial convention [37]. Figs. 7a, 7b, and 7c show the observed, demigrated, and deblurred data of the 100th CSG, respectively. Fig. 8a, 8b, and 8c show the RTM image, singlestep LSRTM image with trace-by-trace consideration, and single-step LSRTM image with beam-by-beam consideration, respectively. We observe that both Figs. $8 \mathrm{~b}$ and $8 \mathrm{c}$ have better imaging resolution and more balanced illumination than Fig. 8a. For detailed demonstration, we zoom-in three parts between CDP [100, 800] and depth [1.25 km, $3.125 \mathrm{~km}]$ from Fig. 8 to show in Fig. 9. From them, we observe that Fig. 9c has the best imaging quality in terms of high resolution and balanced amplitude, especially for the reflectors indicated by the arrows around the overthrust structure.

\section{CONClusions}

LSRTM works well in improving the RTM imaging qualities through iterative linearized inversion with more expensive 

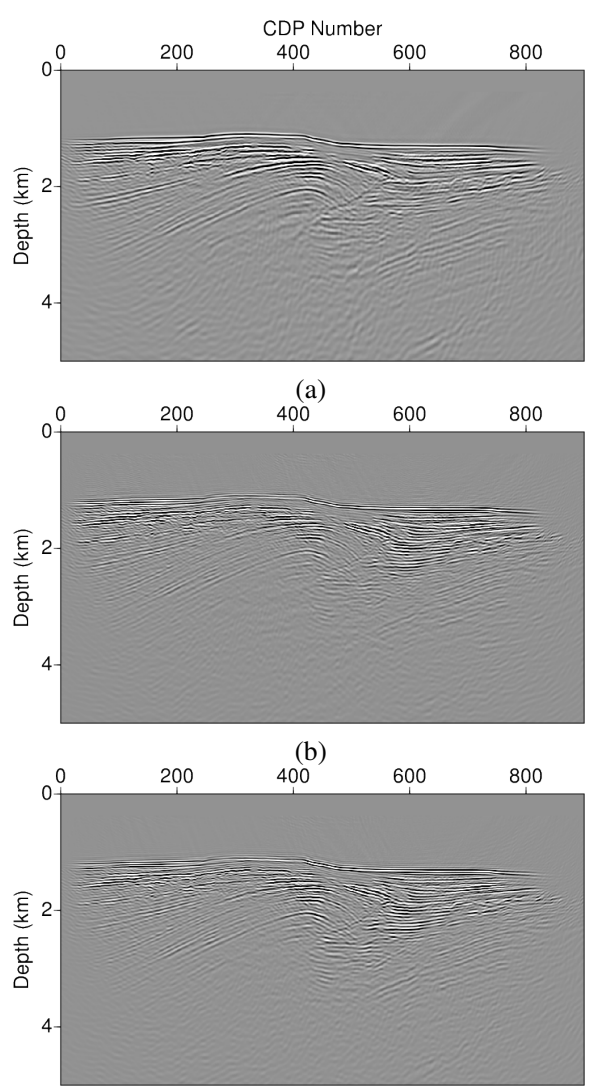

(c)

Fig. 8. Stacked marine images by (a) RTM, single-step LSRTMs with (b) trace-by-trace and (c) beam-by-beam considerations, respectively. We can see that (b) and (c) have better imaging quality than (b).

computational costs. We design adaptive matching filters to precondition the observed data to mitigate the blurring effect caused by the Hessian efficiently. A single-step data-domain LSRTM with trace-by-trace consideration was proposed to improve its efficiency. However, this consideration is not enough for seismic waves, which propagate more likely in beams rather than in rays. Thus, in this study, we propose the single-step data-domain LSRTM with beam-by-beam consideration. To validate the advantage of beams over rays in our research, we introduce a concept named "trace-spread function", which measures the spreading effects of one seismic trace or beam over the migration/demigration processes. We notice that the beam consideration outperforms the trace one with nearly fixed bandwidth and fewer trade-offs in TSF testings. Based on this fact, we approach the beam-by-beam single-step LSRTM at two levels. At the beam level, we have the diagonal consideration to $\mathbf{L L}^{T}$. Each beam consists of a bundle of traces. For the traces inside one beam, we fully consider the deblurring process with the Sherman-Morrison formula. The deblurred beams are then superimposed to form a deblurred data for remigration. Numerical examples show that our method provides promising results of higher resolution and more balanced amplitude over the trace-by-trace singlestep LSRTM and conventional RTM. Here, we only consider the noise-free or less-noisy data. To handle the noisy data, we may furthermore develop an improved cross-correlation
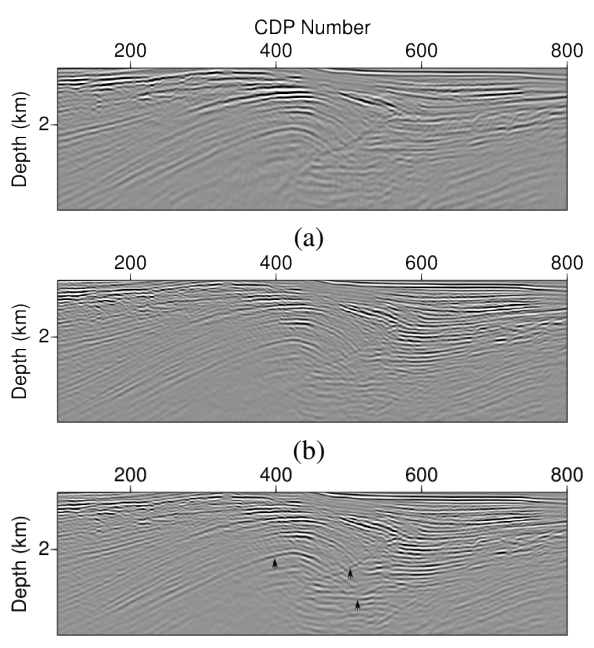

(c)

Fig. 9. (a),(b) and (c): zoom-in views from Figs. 9a, 9b and 9c, respectively. With the trace-by-trace deblurring, (b) outperformed (a). After the bandlimited consideration in beams, (c) even outperforms (b) with higher imaging resolution and more balanced imaging amplitude, especially for the overthrust structures, as marked by the arrows.

objective function-based approach. Even with beam-by-beam consideration, the single-step LSRTM approach presented here can be easily applied to large-scale imaging problems.

\section{APPENDIX A \\ PARAMETERIZATION IN LSRTM}

In the time domain, under the constant-density assumption, the acoustic wave-equation reads

$$
\left(\frac{1}{v(\mathbf{x})^{2}} \frac{\partial^{2}}{\partial t^{2}}-\nabla^{2}\right) p\left(\mathbf{x}, t ; \mathbf{x}_{S}\right)=s\left(t ; \mathbf{x}_{S}\right),
$$

with $v(\mathbf{x})$ being the true velocity model, $p\left(\mathbf{x}, t ; \mathbf{x}_{S}\right)$ the pressure wavefield excited by source $s\left(t ; \mathbf{x}_{S}\right)$ at $\mathbf{x}_{S}$. The initial conditions for solving Eq. (A-1) are $p\left(\mathbf{x}, 0 ; \mathbf{x}_{S}\right)=0$ and $\frac{\partial p\left(\mathbf{x}, 0 ; \mathbf{x}_{S}\right)}{\partial t}=0$. We use the convolutional perfectly matched layers [38] as boundary conditions to mimic wavefield propagation in an infinite space.

We split $v(\mathbf{x})$ into two terms: $v(\mathbf{x})=v_{0}(\mathbf{x})+\delta v(\mathbf{x})$, in which $v_{0}(\mathbf{x})$ is the background velocity and $\delta v(\mathbf{x})$ the velocity perturbation. $v_{0}(\mathbf{x})$ accounts for the kinematic information, and $\delta v(\mathbf{x})$ the reflection waves. We usually call $\delta v(\mathbf{x})$ as the "reflectivity". Expanding the perturbation term $\delta v(\mathbf{x})$ in a Taylor series and dropping the quadratic term at the cost of error $O\left(\delta v(\mathbf{x})^{2}\right)$, we can get

$$
\frac{1}{\left(v_{0}(\mathbf{x})+\delta v(\mathbf{x})\right)^{2}} \approx \frac{1}{v_{0}(\mathbf{x})^{2}}-\frac{2 \delta v(\mathbf{x})}{v_{0}(\mathbf{x})^{3}} .
$$

Substituting Eq. (A-2) into Eq. (A-1) yields

$$
\left\{\begin{array}{l}
\left(\frac{1}{v_{0}(\mathbf{x})^{2}} \frac{\partial^{2}}{\partial t^{2}}-\nabla^{2}\right) p_{0}\left(\mathbf{x}, t ; \mathbf{x}_{S}\right)=s\left(t ; \mathbf{x}_{S}\right), \\
\left.\frac{1}{v_{0}(\mathbf{x})^{2}} \frac{\partial^{2}}{\partial t^{2}}-\nabla^{2}\right) \delta p\left(\mathbf{x}, t ; \mathbf{x}_{S}\right)=\frac{m(\mathbf{x})}{v_{0}(\mathbf{x})^{2}} \frac{\partial^{2} p_{0}\left(\mathbf{x}, t ; \mathbf{x}_{S}\right)}{\partial t^{2}} .
\end{array}\right.
$$

Following [33], we parameterize the logarithmic reflectivity as $m(\mathbf{x})=2 \delta v(\mathbf{x}) / v_{0}(\mathbf{x})$. 
The first equation in Eq. (A-3) propagates the background source wavefield. The second one in Eq. (A-3) accounts for the scattering wavefields inspired by the fictitious source $\frac{m(\mathbf{x})}{v_{0}(\mathbf{x})^{2}} \frac{\partial^{2} p_{0}\left(\mathbf{x}, t ; \mathbf{x}_{S}\right)}{\partial t^{2}}$. We need two modelings in the demigration process by Eq. (A-3): one for $p_{0}$ and the other for $\delta p . \delta p$ is driven by $p_{0}$. For simplification, we express the demigration process in Eq. (A-3) with a compact matrix form:

$$
\mathbf{L m}=\mathbf{d},
$$

with $\mathbf{L}$ being the forward operator, $\mathbf{m}$ the reflectivity model, and $\mathbf{d}$ the seismograms from $\mathbf{m}$. According to the adjoint method [33], we obtain the gradient by applying the following imaging condition:

$$
m_{\text {mig }}(\mathbf{x})=\int_{0}^{T}-\frac{\partial^{2} p_{0}}{v_{0}^{2} \partial t^{2}} \widehat{p} d t,
$$

where $p_{0}$ is the forward propagated source wavefield, and $\widehat{p}$ the adjoint wavefield governed by the following equation:

$$
\left(\frac{\partial^{2}}{v_{0}^{2}(\mathbf{x}) \partial t^{2}}-\nabla^{2}\right) \widehat{p}\left(\mathbf{x}, t ; \mathbf{x}_{S}\right)=\Delta d\left(\mathbf{x}_{R}, t ; \mathbf{x}_{S}\right),
$$

with $\Delta d\left(\mathbf{x}_{R}, t ; \mathbf{x}_{S}\right)$ being the data residual at receiver $\mathbf{x}_{R}$ of shot $\mathbf{x}_{S}$.

\section{ACKNOWLEDGMENT}

The authors greatly thank S. Operto for inspiration of the beam-by-beam consideration. The authors also thank G. Schuster and D. Peter for helpful discussions. The research reported in this publication was supported by King Abdullah University of Science and Technology (KAUST).

\section{REFERENCES}

[1] E. Baysal, D. D. Kosloff, and J. W. Sherwood, "Reverse time migration," Geophysics, vol. 48, no. 11, pp. 1514-1524, 1983.

[2] N. Whitmore, "Iterative depth migration by backward time propagation," SEG Technical Program Expanded Abstracts 1983, pp. 382-385, 1983.

[3] C. J. Leuschen and R. G. Plumb, "A matched-filter-based reversetime migration algorithm for ground-penetrating radar data," IEEE Transactions on Geoscience and Remote Sensing, vol. 39, no. 5, pp. 929-936, 2001.

[4] F. Foroozan and A. Asif, "Time-reversal ground-penetrating radar: Range estimation with cramér-rao lower bounds," IEEE Transactions on Geoscience and Remote Sensing, vol. 48, no. 10, pp. 3698-3708, 2010.

[5] M. E. Yavuz and F. L. Teixeira, "Frequency dispersion compensation in time reversal techniques for uwb electromagnetic waves," IEEE Geoscience and Remote sensing letters, vol. 2, no. 2, pp. 233-237, 2005.

[6] P. Kosmas and C. M. Rappaport, "A matched-filter fdtd-based time reversal approach for microwave breast cancer detection," IEEE Transactions on Antennas and Propagation, vol. 54, no. 4, pp. 1257-1264, 2006.

[7] Z. Wang, H. Ding, G. Lu, and X. Bi, "Reverse-time migration based optical imaging," IEEE Transactions on medical imaging, vol. 35, no. 1, pp. 273-281, 2016.

[8] W. Symes, "The seismic reflection inverse problem," Inverse problems, vol. 25 , no. 12 , p. 123008,2009

[9] I. Lecomte, "Resolution and illumination analyses in psdm: A ray-based approach," The Leading Edge, vol. 27, pp. 650-663, 2008.

[10] P. Lailly, "The seismic inverse problem as a sequence of before stack migrations," Conference on Inverse Scattering, 1983.

[11] G. T. Schuster, "Least-squares cross-well migration," 63rd Annual International Meeting, SEG, Expanded Abstracts, pp. 110-113, 1993.

[12] T. Nemeth, C. Wu, and G. T. Schuster, "Least-squares migration of incomplete reflection data," Geophysics, vol. 61, no. 1, pp. $208-221$, 1999.
[13] H. Kuehl and M. Sacchi, "Robust avp estimation using least-squares wave-equation migration," 72nd Annual International Meeting, SEG, Expanded Abstracts, pp. $281-284,2002$.

[14] M. L. Clapp, R. G. Clapp, and B. L. Biondi, "Regularized least-squares inversion for 3-d subsalt imaging," 75th Annual International Meeting, SEG, Expanded Abstracts, pp. 1814-1817, 2005.

[15] J. Wang, H. Kuehl, and M. D. Sacchi, "High-resolution wave-equation ava imaging: Algorithm and tests with a data set from the western canadian sedimentary basin," Geophysics, vol. 70, no. 5, pp. S91-S99, 2005.

[16] Y. Tang, "Target-oriented wave-equation least-squares migration/inversion with phase-encoded hessian," Geophysics, vol. 74, no. 6, pp. WCA95-WCA107, 2009.

[17] M. Wong, S. Ronen, and B. Biondi, "Least-squares reverse time migration/inversion for ocean bottom data: a case study," SEG Technical Program Expanded Abstracts, pp. 2369-2373, 2011.

[18] W. Dai and G. T. Schuster, "Plane-wave least-squares reverse-time migration," Geophysics, vol. 78, no. 4, pp. S165-S177, 2013.

[19] M. Wong, B. Biondi, and S. Ronen, "Imaging with primaries and free-surface multiples by joint least-squares reverse time migration," Geophysics, vol. 80, pp. S223-S235, 2015.

[20] Y. Zhang, L. Duan, and Y. Xie, "A stable and practical implementation of least-squares reverse time migration," Geophysics, vol. 80, no. 1, pp. V23-V31, 2015.

[21] G. Yao and H. Jakubowicz, "Least-squares reverse-time migration in a matrix-based formulation," Geophys. Prospect., vol. 64, pp. 611-621, 2016.

[22] Y. Liu, J. Teng, T. Xu, Z. Bai, H. Lan, and J. Badal, "An efficient step-length formula for correlative least-squares reverse time migration," Geophysics, vol. 81, no. 4, pp. S221-S238, 2016.

[23] X. Liu, Y. Liu, H. Lu, H. Hu, and M. Khan, "Prestack correlative leastsquares reverse time migration," Geophysics, vol. 82, no. 2, pp. S159S172, 2017

[24] L. Xu and M. D. Sacchi, "Preconditioned acoustic least-squares twoway wave-equation migration with exact adjoint operator," Geophysics, vol. 83, no. 1, pp. S1-S13, 2017.

[25] R. E. Plessix and W. A. Mulder, "Frequency-domain finite-difference amplitude-preserving migration," Geophys. J. Int., vol. 157, pp. 975987, 2004

[26] A. Guitton, "Amplitude and kinematic corrections of migrated images for nonunitary imaging operators," Geophysics, vol. 69, pp. 1017-1024, 2004.

[27] K. Peacock and S. Treitel, "Predictive deconvolution: Theory and practice," Geophysics, vol. 34, no. 2, pp. 155-169, 1969.

[28] S. Rajagopalan and P. Milligan, "Image enhancement of aeromagnetic data using automatic gain control," Exploration Geophysics, vol. 25 , no. 4, pp. 173-178, 1994

[29] Q. Liu and D. Peter, "One-step data-domain least-squares reverse-time migration," Geophysics, vol. 83, no. 4, pp. 1-46, 2018.

[30] N. R. Hill, "Prestack gaussian-beam depth migration," Geophysics, vol. 66, no. 4, pp. 1240-1250, 2001.

[31] W. W. Hager, "Updating the inverse of a matrix," SIAM review, vol. 31, no. 2, pp. 221-239, 1989.

[32] S. Fomel and J. Claerbout, "Streaming prediction-error filters," in $S E G$ Technical Program Expanded Abstracts 2016. Society of Exploration Geophysicists, 2016, pp. 4787-4791.

[33] J. Tromp, C. Tape, and Q. Liu, "Seismic tomography, adjoint methods, time reversal, and banana-donut kernels," Geophys. J. Int., vol. 160, pp. 195-216, 2005.

[34] J. Hu, G. Schuster, and P. A.Valasek, "Poststack migration deconvolution," Geophysics, vol. 66, pp. 939 - 952, 2001.

[35] M. Sambridge and B. Kennett, "Boundary value ray tracing in a heterogeneous medium: a simple and versatile algorithm," Geophysical Journal International, vol. 101, no. 1, pp. 157-168, 1990.

[36] J. Virieux, "P-SV wave propagation in heterogeneous media: velocitystress finite-difference method," Geophysics, vol. 51, pp. 889-901, 1986.

[37] Q. Liu, J. Zhang, and H. Zhang, "Eliminating the redundant source effects from the cross-correlation reverse-time migration using a modified stablized division," Computers Geosciences, vol. 92, pp. 49-57, 2016.

[38] D. Komatitsch and R. Martin, "An unsplit convolutional perfectly matched layer improved at grazing incidence for the seismic wave equation," Geophysics, vol. 72, pp. SM155-SM167, 2007. 